\title{
CONTROL BIOLÓGICO DEL OÍDIUM POR ACCIÓN DE Trichoderma Harzianum $Y T$. Sp. TL. EN PLANTAS DE VID Vitis Vinifera A NIVEL DE CAMPO, EN POCOLLAY -TACNA
}

Responsable: MSc. Daladier Castillo Cotrina Miembro: Mblgo. César Huanacuni Lupaca

\begin{abstract}
RESUMEN
El presente trabajo tuvo como objetivo determinar el grado de control de dos hongos de Trichoderma sobre el Oidium en los racimos de las plantas de vid. Se aplicó $10 \mathrm{ml}$ de inóculo de Trichoderma , constituido por una suspensión densa de conidios, por aspersión sobre los racimos de las plantas de vid de las 05 repeticiones por tratamiento.

Se determinó que $T$. sp TL fue el que presentó el mayor grado de control sobre el Oidíum y el menor T. harzianum con valores de 06 y 05 respectivamente en función de la disminución de infección de Oidium en los racimos de uva de las plantas de vid.
\end{abstract}

\begin{abstract}
The grade of control for two Trichoderma on the oidium on berry clusters have been studies. The application of Trichoderma was made inoculating $10 \mathrm{ml}$ of conidia suspended on the berry clusters of the five repetition for treatment.

It was determined that T.sp TL had better control grade on oidium and the less $T$. harzianum, with values of 06 and 05 respectivament in function of the reduction of the oidium infection in the berry cluster.
\end{abstract}

\section{INTRODUCCION}

Trichoderma es utilizado como biocontrolador de agentes etiológicos de enfermedades en diferentes cultivos vegetales (10). Su acción biocontroladora guarda relación con la acción antagónica que presenta contra muchos agentes de enfermedades de plantas, tales como las causadas por hongos como el Oidium (3). Es un hongo que presenta varias especies, algunas de ellas son $T$. harzianum, $T$. aureoviride, que se desarrollan sobre medios artificiales como el agar papa sacarosa, agar papa dextrosa, agar extracto de malta $2 \%(8)$ y a temperaturas mesófilas como el de $27^{\circ} \mathrm{C}$, en presencia de oxígeno luz (3). Macroscópicamente originan de manera general una colonia micelial, blanca al inicio y verde posteriormente, etapa coincidente con la fase esporulación. (7)(8).

Trichoderma para efectuar el biocontrol podría hacer uso de los diferentes mecanismos de acción que exhibe; puede producir antibióticos, es capaz de competir por nutrientes y puede ejercer micoparasitismo que en general consta de cuatro etapas: 1) crecimiento quimiotrópico de Trichoderma, 2) reconocimiento del hospedante por el micoparásito, 3) secreción de enzimas extracelulares (B-1,3 glucanas, quitinasa y celulasa), 4) Lisis del hospedante (1).

Podemos describir a la vid como una planta trepadora, vivaz, de tronco retorcido y largos sarmientos. Sus hojas son grandes y pecioladas, están divididas en cinco lóbulos más o menos profundos; frente a algunas de ellas nace un zarcillo o tijerilla que puede enroscarse a cualquier cosa. Las flores se agrupan en panícula, también opuestas a las hojas; cada flor consta de cáliz, corola, estambres y un pistilo. El fruto es una baya, la uva, ésta encierra las semillas. Las variedades se diversifican por la forma de sus hojas y, principalmente, por la característica del fruto (2).

El Oidium, una enfermedad que usualmente se presenta en la vid, tiene como agente causante al hongo Uncinula necator (Schw) Burr, cuyo micelio (talo) es enteramente superficial.

Los sintomas del Oidium se presentan atacando a los órganos jóvenes de la vid, hojas, brotes, sarmientos, inflorescencias y frutos, manifestándose en forma de manchas blancas, que se cubren de una capa blanquecina y pulverulenta; que al final de la actividad de la planta; cambió a color bruno (marrón) (2).

Las pruebas experimentales de antagonismo entre dos hongos (crecimiento dual) nos da el indicativo del grado de control que puede ejercer el hongo que se pretende utilizar en el campo para el biocontrol sobre el hongo considerado fitopatógeno. Estas pruebas pueden realizarse sembrando a los hongos a evaluar sobre la superficie de una planta de vid y midiendo luego el radio de crecimiento de ambos y de invasión de uno sobre el otro (4) (11).

En Tacna, el cultivo de la vid ha ido incrementándose en los últimos años, haciéndose necesario también un apoyo técnico 
en esta área, para llevar el cultivo de la mejor manera y obtener una abundante producción y de calidad, adecuándolas tanto para su consumo en mesa como para su industrialización en vinos, piscos y derivados (9).

Con la finalidad de incentivar y apoyar al desarrollo de la viticultura en el departamento de Tacna, es importante la realización de trabajos de investigación destinados a mejorar la calidad de las uvas en nuestra zona (2).

El Oidium es causante del deterioro y disminución de la producción de uva en los viñedos de Tacna. La información que se tiene sobre el uso de métodos biológicos utilizando antagonistas contra el Oidium es reducida; por el momento se tiene el reporte del grado de control que ejercieron los dos Trichoderma que son parte del proyecto actual y un tercer Trichoderma que no ejerció control sobre el Oidíum de los racimos de la vid, pero cuyas aplicaciones se hizo cuando los racimos de uva ya estaban formados e infectados con el Oidium (5).

El objetivo general del trabajo fue: determinar el grado de control del Oidium en los racimos de las plantas de Vitis vinifera por la acción de Trichoderma a nivel de campo en Pocollay - Tacna. Los objetivos específicos fueron: 1) Determinar el grado de control de Trichoderma harzianum y Trichoderma sp. TL . sobre el Oidium en los racimos de vid; 2) Establecer cuál de los Trichoderma a evaluar presenta mayor grado de control sobre el Oidium en los racimos de vid.

\section{MATERIALYMÉTODOS}

1. Material Biológico: Cultivos de Trichoderma: $T$. harzianum (cultivo no nativo, procedente del SENASA de Lima) y $T$. sp. TL (cultivo nativo). Plantas de cultivo de vid "Vitis vinifera" del campo de "Sobraya", Pocollay.

2. Variables de Estudio: Variables Dependientes (v.d.): grado de control, por separado, de $T$. harzianum y $T$. sp. TL sobre el Oidium en los racimos de la planta de vid; Variable Independiente (v.i.): racimos de las plantas de vid. Indicadores de la V.i. : 1) Nivel de infección del grano de uva con Oidium; 2) Volumen del racimo, del escobajo del racimo, del total de granos de uva del racimo, del total de granos con superficie lisa del racimo y del total de granos con superficie rugosa del racimo; 3 ) Número del total de granos de uva de tamaños grande, mediano y pequeño en el racimo.

3. Tratamientos: Se consideraron para la experimentación 05 tratamientos, cada tratamiento con 05 repeticiones, cada repetición con una unidad experimental, constituida por un racimo de uva. Los tratamientos fueron:

Tratamientos Experimentales (T.E. = 02): 1) T.E. $1=T$. harzianum + racimo de uva de plantas de vid ; 2 ) T.E. $2=T$. sp TL + racimo de uva de plantas de vid.

Tratamientos Controles (T.C. $=02$ ): 1) T.C. = racimo de uva con Oidium; 2) T.C. $+=$ racimo de uva sin Oidium.

4. Lugar de Experimentación: La experimentación se realizó en el fundo "Sobraya" en Pocollay - Tacna-Perú, que mantiene el cultivo de la vid desde hace algunos años atrás.

5. Plantas de Vid Empleados en la Experimentación: Las plantas de vid para la experimentación fueron elegidas al azar del total de plantas del cultivo en el inicio de la fase de brotamiento (junio del 2007). Las plantas elegidas conjuntamente con las demás plantas del cultivo fueron cuidadas para su crecimiento y desarrollo realizando deshierbos periódicos, fertilización y riegos por inundación para la mantención de la humedad necesaria para las plantas de vid.

Cuando las plantas de vid estuvieron en la fase inicial de formación de fruto, a aquellas plantas que inicialmente se las había separado para que formaran parte de los tratamientos experimentales se las aplicó el Trichoderma ; a las demás plantas que también previamente se las habia separado para que formaran parte de los tratamientos controles positivos se les aplicó el azufre, que es el que prevee la no aparición del Oidium, y a las que estaban separadas como tratamientos controles negativos no se les aplicó nada, es decir ni azufre ni Trichoderma.

El azufre aplicado a las plantas de vid de los tratamientos correspondientes se hizo del mismo modo y en una sola vez como se hizo con todas las plantas de vid del campo de cultivo.

6.Preparación de los Inóculos de Trichoderma para su Aplicación Contra el Oidium en la Vid: Se obtuvo una suspensión densa de conidios de Trichoderma de color verdoso colocando inóculos de micelio, que fueron extraídos con una asa de Koll del cultivo stock del hongo, en viales conteniendo $1 \mathrm{ml}$ de solución salina fisiológica $0,85 \%$ y que fueron agitados fuertemente. Se sembró 
$0,1 \mathrm{ml}$ de la suspensión densa de conidios, por encamada, en una placa Petri conteniendo Agar papa Dextrosa $2 \%$. La placa Petri luego fue llevada a incubación a $25^{\circ} \mathrm{C}$ durante 7 días (tiempo en el que se produjeron abundantes conidios que se ven de un color verdoso sobre la superficie del medio de cultivo en la placa Petri).

Se cosecharon los conidios de Trichoderma de la placa Petri colocando sobre el micelio esporulado $05 \mathrm{ml}$ de solución salina fisiológica $0,85 \%$ con 30 perlas de vidrio y haciendo movimientos de rotación a uno y otro lado hasta que se obtuvo una suspensión densa de los conidios del hongo. La suspensión de conidios fue vertida posteriormente a un frasco de boca ancha. En este frasco de boca ancha en total se le añadió la suspensión de conidios de dos placas Petri, por lo cual en este frasco en total se agregó $10 \mathrm{ml}$ de suspensión densa de conidios. Finalmente al frasco se le adicionó y mezcló homogéneamente $1 \mathrm{ml}$ al $10 \%$ de la sustancia adherente PEGASOL. Este frasco con los $10 \mathrm{ml}$ de la suspensión de conidios constituyó el inóculo para las 05 repeticiones de un tratamiento.

\section{Aplicación del Inóculo de Trichoderma A} las Plantas y Racimos de Vid: El inóculo constituído por los $10 \mathrm{ml}$ de suspensión densa de conidios fue vertido desde el frasco, donde estuvo contenido, hacia el recipiente asperjador; con el cual luego se asperjó, bañando totalmente los 05 racimos de uva, unidades experimentales, que fueron las 05 repeticiones de un tratamiento. Se emplearon 02 asperjadores considerando que fueron 02 Trichoderma los empleados para la experimentación.

La aplicación del Trichoderma a las plantas de vid que constituyeron los tratamientos experimentales se hizo desde su inicio de formación de fruto, y en la que en ningún racimo fue observada la presencia del Oidium, repitiendo la aplicación periódicamente del Trichoderma hasta el tiempo en que los racimo de vid estuvieron cerca de la cosecha: bajo el siguiente orden: 1ra. aplicación: 05 de agosto; 2da. Aplicación 30 de setiembre; 3ra. aplicación: 14 de octubre; 4ta. aplicación: 04 de noviembre; 5 ta. aplicación: 02 de diciembre; $6 \mathrm{ta}$. aplicación: 23 de diciembre; $7 \mathrm{ma}$. aplicación: 20 de enero; $8 \mathrm{va}$. aplicación: 09 de marzo; 9na. aplicación: 09 de marzo; 10ma. evaluación: 13 de abril.
8. Mantenimiento y Evaluación de las Plantas de Vid de la Experimentación: El terreno donde estuvieron las plantas de vid fue deshierbado y regado por inundación periódicamente, para mantener limpio y con la humedad necesaria para las plantas de vid, hasta dos meses antes de cosechar la uva, coincidente con la madurez comercial de la uva. La evaluación de los racimos de uva se hizo coincidentemente con el tiempo de cosecha que fue el 13 abril de 2008 ( 10,5 meses después de su inicio de la fase de brotamiento y 8,5 meses después de la primera aplicación de Trichoderma ). Los racimos de uva de la experimentación fueron desprendidos de sus respectivas plantas de vid y trasladados al laboratorio de Micología-Virología para el análisis respectivo. En el laboratorio se evaluó cada racimo de uva de los tratamientos, con los siguientes parámetros:

- Nivel de infección con Oidíum en los granos de uva del racimo de vid; fue determinado por observación macroscópica y según una escala de valores de 0 a 19 propuesto por el autor, que fue el siguiente:

Nivel de Infección (N.I.) con Oidium y Características del Mismo en los Racimos de las Plantas de Vid.

\begin{tabular}{|l|}
\hline N.I. Caracteristicas \\
\hline - Todo el racimo de uva no presenta sintomas o signos \\
de Oidium. \\
\hline - Alrededor de la $1 / 4$ parte del número de los granos de \\
uva del racimo de uva presentan Oidium con poco \\
micelio sobre la superficie del grano de uva infectado \\
y con una superficie lisa. \\
\hline - Alrededor de la $1 / 4$ parte del número de los granos de \\
uva del racimo de uva presentan Oidium con \\
abundante micelio sobre la superficie del grano de \\
uva infectado y con una superficie lisa. \\
\hline - Alrededor de la $1 / 4$ parte del número de los granos de \\
uva del racimo de uva presentan Oidium con poco o \\
abundante micelio sobre la superficie del grano de \\
uva infectadoy con una superficie rugosa. \\
\hline -Alrededor de la mitad del número de los granos de uva \\
del racimo de uva presentan Oidium con poco micelio \\
sobre la superficie del grano de uva infectado y con \\
una superficie lisa. \\
\hline - Alrededor de la mitad del número de los granos de \\
uva del racimo de uva presentan Oidium con \\
abundante micelio sobre la superficie del grano de \\
uva infectado y con una superficie lisa. \\
\hline - Alrededor de la mitad del número de los granos de \\
uva del racimo de uva presentan Oidium con poco o \\
abundante micelio sobre la superficie del grano de \\
uva infectado y con una superficie rugosa. \\
\hline - Alrededor de las $3 / 4$ partes del número de los granos \\
de uva del racimo de uva presentan Oidium con poco \\
micelio sobre la superficie del grano de uva infectado \\
y con una superficie lisa. \\
\hline -Alrededor de las $3 / 4$ partes del nümero de los granos \\
de uva del racimo de uva presentan Oidium con \\
abundante micelio sobre la superficie del grano de \\
uva infectado y con una superficie lisa. \\
\hline - Alrededor de las $3 / 4$ partes del número de los granos de \\
uva del racimo de uva presentan Oidium con poco o \\
\hline
\end{tabular}


abundante micelio sobre la superficie del grano de uva infectado y con una superficie rugosa.

-Todo el racimo de uva presenta Oidium con poco micelio sobre la superficie del grano de uva infectado y con el $100 \%$ de granos con superficie lisa y $0 \%$ de granos con superficie rugosa.

-Todo el racimo de uva presenta Oidium con poco micelio sobre la superficie del grano de uva infectado y con el $75 \%$ de granos con superficie lisa y $25 \%$ de granos con superficie rugosa.

-Todo el racimo de uva presenta Oidium con poco micelio sobre la superficie del grano de uva infectado y con el $50 \%$ de granos con superficie lisa y $50 \%$ de granos con superficie rugosa.

-Todo el racimo de uva presenta Oidíum con poco micelio sobre la superficie del grano de uva infectado y con el $25 \%$ de granos con superficie lisa y $75 \%$ de granos con superficie rugosa.

-Todo el racimo de uva presenta Oidium con poco micelio sobre la superficie del grano de uva infectado y con el $0 \%$ de granos con superficie lisa y $100 \%$ de granos con superficie rugosa.

-Todo el racimo de uva presenta Oidium con abundante micelio sobre la superficie del grano de uva infectado y con el $100 \%$ de granos con superficie lisa y $0 \%$ de granos con superficie rugosa.

- Todo el racimo de uva presenta Oidium con abundante micelio sobre la superficie del grano de uva infectado y con el $75 \%$ de granos con superficie lisa y $25 \%$ de granos con superficie rugosa.

- Todo el racimo de uva presenta Oidium con abundante micelio sobre la superficie del grano de uva infectado y con el $50 \%$ de granos con superficie lisa y $50 \%$ de granos con superficie rugosa.

- Todo el racimo de uva presenta Oidium con abundante micelio sobre la superficie del grano de uva infectado y con el $25 \%$ de granos con superficie lisa y $75 \%$ de granos con superficie rugosa.

-Todo el racimo de uva presenta Oidíum con poco micelio sobre la superficie del grano de uva infectado y con el $0 \%$ de granos con superficie lisa y $100 \%$ de granos con superficie rugosa.

Macroscópicamente se consideró que los granos de uva estaban infectados con Oidium cuando se observó que sobre la superficie de estos granos había una especie de polvillo o lanilla de color plomizo amarronado. Microscópicamente se comprobó que estos signos correspondieron al Oidium cuando se realizó preparados en fresco.

- Volumen del racimo de vid. Fue determinado sumergiendo el racimo de uva en agua contenida en un recipiente, que por rebose el agua desplazada fue recibida en un segundo recipiente que contenia al primero. Esta agua fue medida en una probeta y esto constituyó el volumen del racimo de vid.

- Volumen total de granos de uva del racimo. Fue medido de manera similar al del volumen del racimo de vid.

- Volumen del escobajo del racimo de vid. Fue determinado por diferencia del volumen del racimo de vid con el volumen del total de los granos de uva del racimo de vid correspondiente.

- Volumen total de granos de uva con superficie lisa del racimo de vid. Fue determinado de manera similar al del volumen del racimo de vid

- Volumen total de granos de uva con superficie rugosa del racimo de vid. Fue establecido por diferencia entre el Volumen del total de granos de uvas del racimo y el volumen del total de granos.

- Número total de granos de uva con superficie lisa en el racimo de vid. Fue establecido por conteo individual de entre el total de los granos de uva desprendidos del racimo de vid.

- Número total de granos de uva con superficie rugosa en el racimo de vid. Fue determinado por diferencia entre el número del total de granos de uva en el racimo de vid y el número total de granos de uva con superficie lisa del mismo racimo de vid.

- Número de granos de uva en el racimo de vid de tamaño pequeño (longitud menos de 0.8 $\mathrm{cm}$ ), mediano (longitud entre 0,8 y $1,2 \mathrm{~cm}$ ) y grande (longitud mayor de $1,2 \mathrm{~cm}$ ). Fue determinado midiendo la longitud del grano de uva con una regla milimetrada y según su medición clasificado en los rangos de longitud de pequeño, mediano y grande y luego contados los granos individualmente para cada grupo de tamaño.

- Número de granos de uva con superficie lisa en el racimo de vid de tamaño pequeño ( longitud menos de $0.8 \mathrm{~cm}$ ). mediano ( longitud entre 0,8 y $1,2 \mathrm{~cm}$ ) y grande (longitud mayor de $1,2 \mathrm{~cm}$ ). Fue determinado sólo para los granos de uva con superficie lisa, de manera similar a lo que se hizo para determinar el número total de granos de uva en el racimo de vid, de tamaño pequeño, mediano y grande.

En la determinación del valor de un parámetro en un tratamiento, que es el que se muestra en los resultados, éste se obtuvo promediando los valores de las 05 repeticiones del tratamiento.

9.Determinación del Grado de Control de Trichoderma sobre el Oidium en los Racimos de las Plantas de Vid: Se consideró que hubo control del Oidíum por Trichoderma cuando los valores determinados en los diferentes parámetros evaluados estuvieran entre los valores de los correspondientes parámetros de evaluación de los tratamientos controles negativo ( TC- = infectado con Oidium) y positivo ( $\mathrm{TC}+=$ no infectado con Oidium).

El valor del grado de control, para los demásparámetros, se determinó : para el caso del nivel de infección con Oidium, en función del valor disminuido de infección que se obtuvo por diferencia de los valores del nivel de infección entre los tratamientos; en el caso de los demás parámetros el grado de control se expresó en términos porcentuales, que se obtuvo por diferencia entre los valores de los tratamientos experimentales con el tratamiento control negativo teniendo en cuenta a su vez que la diferencia de 


\section{III.RESULTADOS}

Cuadro 1: Nivel de infección por Oidium de los racimos de vid de cada tratamiento de la experimentación.

\begin{tabular}{|l|l|}
\hline TRATAMIENTO & NIVEL DE INFECCION \\
\hline TE1 & 12 \\
TE2 & 11 \\
\hline TC- & 17 \\
TC + & 00 \\
\hline
\end{tabular}

TE1=Trat. experimental 2=Trichoderma harzianum + Racimo de vid con Oidium; TE2=Trat. Experimental 3=Trichoderma sp. TL + Racimo de vid con Oidium; TC$=$ Trat. Control $-\quad=$ Racimo de vid con Oidium; TC+=Trat. Control + = Racimo de vid sin Oidium .

Cuadro 2: Grado de control de Trichoderma sobre el Oidíum en función del nivel de infección por Oidium en los racimos de vid de cada tratamiento de la experimentación obtenido por diferencia del valor de infección del tc- con el te.

\begin{tabular}{|l|l|}
\hline TRATAMIENTO & GRADO DE CONTROL \\
\hline TE1 & 05 \\
TE2 & 06 \\
\hline
\end{tabular}

TE1 $=$ Trat. experimental $2=$ Trichoderma harzianum + Racimo de vid con Oidium; TE2=Trat. Experimental 3=Trichoderma sp. TL + Racimo de vid con Oidium; TC$=$ Trat. Control $\mathrm{-}=$ Racimo de vid con Oidium .

Cuadro 3. Volumen $\left(\mathrm{cm}^{3}\right)$ por racimo de vid del: racimo total, escobajo, granos de uva total, granos de uva con superficie lisa (gsl) y de granos de uva con superficie rugosa (gsr) de cada tratamiento de la experimentación.

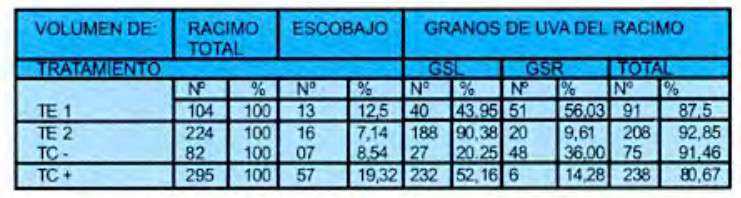

TE1=Trat. experimental 2 =Trichoderma harzianum + Racimo de vid con Oidium; TE2=Trat. Experimental 3=Trichoderma sp. TL + Racimo de vid con Oidium; TC$=$ Trat. Control $-=$ Racimo de vid con Oidium; TC+=Trat. Control $+=$ Racimo de vid sin Oidium.

Cuadro 4: Grado de control (\%) de Trichoderma sobre el Oidium en función del incremento de volumen $\left(\mathrm{cm}^{3}\right)$ por racimo del: racimo total, escobajo, granos de uva total, y con superficie lisa (gsl) de cada tratamiento de la experim. considerando un valor porcentual a la diferencia de volumen de los t.e. Con el t.c- teniendo como referencia de $100 \%$ de control a la diferencia de volumen del tc+ con el del tc- $\left(t^{+}+t^{+}\right)$.

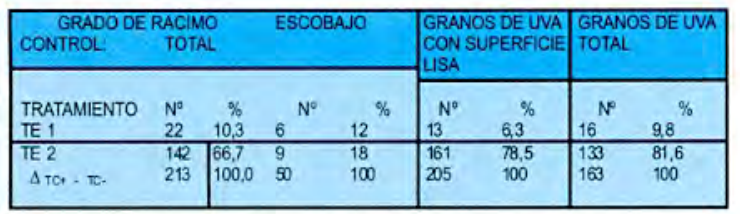

Te1 =Trat. experimental 2 =Trichoderma harzianum + Racimo de vid con Oidium; TE2=Trat. Experimental $3=$ Trichoderma sp. TL+Racimo de vid con Oidium.
Cuadro 5: Número de granos de uva (ngu) total por racimo de tamaño pequeño $(p=$ menor de $0,8 \mathrm{~cm})$, mediano $(\mathrm{m}=1,2-0,8 \mathrm{~cm})$ y grande $(\mathrm{g}=$ mayor de $1,2 \mathrm{~cm})$ para cada tratamiento de la experimentación.

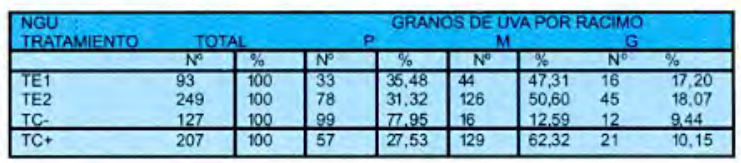

Te1 $=$ Trat. experimental $2=$ Trichoderma harzianum + Racimo de vid con Oidium; TE2=Trat. Experimental $3=$ Trichoderma sp. TL+ Racimo de vid con Oidium; TC$=$ Trat. Control $-=$ Racimo de vid con Oidium; TC+=Trat. Control $+=$ Racimo de vid sin Oidium .

Cuadro 6: Grado de control (\%) de Trichoderma sobre el Oidium en funcion del incremento del número de granos de uva total por racimo y según el tamaño predominante (mediano) dentro de los tipos de pequeño $(p=$ menor de $0,8 \mathrm{~cm})$, mediano $(\mathrm{m}=1,2-0,8 \mathrm{~cm})$ y grande $(\mathrm{g}=$ mayor de $1,2 \mathrm{~cm})$ para cada tratamiento de la experimentación considerando un valor porcentual a la diferencia del número de granos de uva de los t.e. con el t.c- teniendo como referencia de $100 \%$ de control a la diferencia del número de granos de uva del tc+ con el del tc$\left(\mathrm{tc}^{+}-\mathrm{tc}^{-}\right)$.

\begin{tabular}{|c|c|c|c|c|c|c|c|c|}
\hline \multirow{2}{*}{$\begin{array}{l}\text { GRADD DE } \\
\text { CONTROL }\end{array}$} & \multirow{2}{*}{$\begin{array}{l}\text { TOTAL } \\
\mathrm{N}^{\circ}\end{array}$} & \multicolumn{6}{|c|}{ NUMMERO DE GRANOS DEUVA POR RACIMO } & \multirow{2}{*}{${ }^{G}$} \\
\hline & & $\%$ & $N^{0}$ & & $\mathrm{~N}^{\circ}$ & & $\mathrm{N}^{0}$ & \\
\hline TE1 & -34 & -425 & -66 & 157,1 & 110 & 97,34 & 4 & 44,4 \\
\hline TE2 & 122 & 152,5 & -21 & 49,9 & 28 & 24,77 & 33 & 366,7 \\
\hline a ra & 80 & 100 & -42 & 100 & 113 & 100 & 9 & 100 \\
\hline
\end{tabular}

TE1 $=$ Trat. experimental $2=$ Trichoderma harzianum + Racimo de vid con Oidium; TE2=Trat. Experimental $3=$ Trichoderma sp. $\mathrm{TL}+$ Racimo de vid con Oidium.

Cuadro 7: Número de granos de uvas con superficie lisa (gsl) por racimo y según los tamaños de pequeño $(p=$ menor de $0,8 \mathrm{~cm})$, mediano $(\mathrm{m}=1,2-0,8 \mathrm{~cm})$ y grande $(\mathrm{g}=$ mayor de $1,2 \mathrm{~cm}$ ) para cada tratamiento de la experimentación.

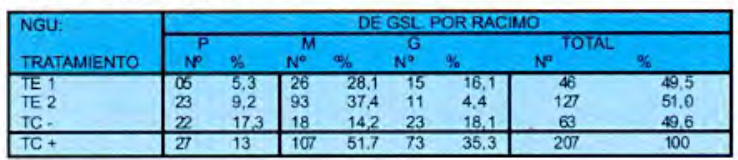

TE1 =Trat. Experimental 2 =Trichoderma harzianum + Racimo de vid con Oidium; TE2=Trat. Experimental $3=$ Trichoderma sp. TL + Racimo de vid con Oidium; TC$=$ Trat. Control $-=$ Racimo de vid con Oidium; TC+=Trat. Control + = Racimo de vid sin Oidium.

Cuadro 8: Grado de control (\%) de Trichoderma sobre el oidium en funcion del incremento del $\mathrm{N}^{\circ}$ de granos de uva con superficie lisa (gsl) por racimo y según el tamaño predominante (mediano) pequeño $(p=$ menor de $0,8 \mathrm{~cm})$, mediano $(\mathrm{m}=1,2-0,8 \mathrm{~cm})$ y grande $(\mathrm{g}=$ mayor de $1,2 \mathrm{~cm}$ ) para cada tratamiento de la experim. considerando un valor porcentual a la diferencia del $\mathrm{N}^{\circ}$ de granos de uva de los t.e. con el t.c- teniendo como referencia de $100 \%$ 
de control a la diferencia del número de granos de uva del tc+ coneldel tc- $($ tot - to $)$.

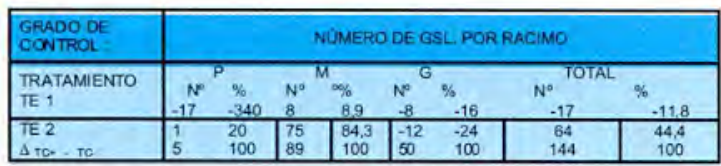

Te1 =Trat. experimental 2 =Trichoderma harzianum + Racimo de vid con Oidium; TE2=Trat. Experimental $3=$ Trichoderma sp. $\mathrm{TL}+$ Racimo de vid con Oidium.

\section{IV.DISCUSIÓN}

El biocontrol encontrado por acción de los Trichoderma sobre el Oidium en los racimos de vid corrobora el conocimiento que se tiene de esta acción de Trichoderma en trabajos anteriores (5).

Se sabe que Trichoderma puede presentar diferencias de acción biocontroladora a diferentes hongos fitopatógenos, diferencias que se dan entre o dentro de las especies de este género; por lo cual esto explica porque Trichoderma sp. TL tuvo un grado de biocontrol diferente al de T. harzianum. (6)(10).

Es probable que el mayor grado de control encontrado en Trichoderma sp. TL sea a que es una especie nativa, propia del lugar de Tacna, en comparación al otro Trichoderma que es proveniente de fuera de Tacna.

\section{CONCLUSIONES}

En las condiciones trabajadas se concluye en lo siguiente:

1-T. sp. TLejerció un grado de biocontrol de 06 . 2-T. harzianum ejerció un grado de biocontrol de 05 .

\section{RECOMENDACION}

Seguir investigando el efecto biovontrolador de Trichoderma sp.TL con aplicaciones periódicas del mismo sobre todo la planta de vid desde el inicio de su etapa vegetativa hasta cerca del término de la fase de maduración del fruto nivel de campo para un mayor control del OIDIUM.

\section{BIBLIOGRAFIA}

Arenas, M.a. 1997. Biocontroladores de Sclerotium cepivorum, berk en cebolla ( A.cepa L.) Cv. Roja arequipeña. Tesis para obtener el título profesional de ingeniero agrónomo. Universidad Nacional San Agustín. Facultad de Ciencias Biológicas y Agropecuarias, Escuela Profesional de Agronomía.

Cutipa, D., Y. 2002. Informe de práctica pre profesional "viticultura aplicada en el fundo monasterio y fundo SOBRAYA". CFAT. Tacna.

Castillo C., D. 2005. Evaluación del crecimiento y desarrollo de Trichoderma auereo viride TAT-1 en una fermentación de estado sólido sobre sustrato de arroz.FACI,COIN,UNJBG. Tacna. Perú.

Castillo C., D. 2001. Optimización de algunos parámetros para la producción de conidios de Trichoderma $s p$ en fermentación de estado sólido. Tesis de maestría, UNT. Trujillo-Perú.

Castillo C., D. Y Cesar Huanacuni L. 2007. Prevención del Oidium en plantas de Vitis vinifera por acción de Trichoderma en Pocollay -Tacna. $\mathrm{FACl}$. COIN. UNJBG. Tacna.

Garcia A.f.a.2001.Antagonismo de Trichoderma aureoviride en el control de Fusarium oxysporum en Allium Cepa L. Tesis. Facultad de Ciencias. U.N.J.B.G. Tacna-Perú.

Mostacero L.,j. Y Col. 1989. Botánica sistemática. Fanerogamia. Facultad de $\quad \mathrm{C}$ i e n c i a s Biológicas, Universidad Nacional de Trujillo. Trujillo-Perú.

Rifai, M.a. 1969. A revision of the genus Trichoderma . Mycological papers $N^{\circ} 116$. Commonwwealth Mycological institute. England.

Sarasola, A.a. y M.rocca de Sarasola.1975. Fitopatología.Tomo I. Edit. Hemisferio Sur. Argentina. 285p.

Stefanova N.,M. 1998. Métodos de aplicación y efectividad de Trochoderma spp. Como antagonista de hongos fitopat'genos. Curso internacional de control biológico BIOSAV-4. Instituto de Investigaciones de Sanidad Vegetal. La Habana-Cuba.

\section{ANEXOS}

\section{Fotos de Racimos de Vid al Final de la Experimentación}

Tratamientos Controles:

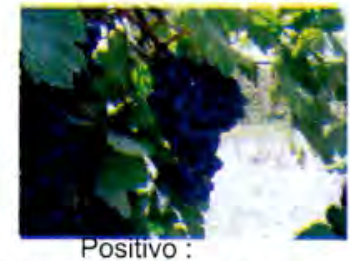

Racimo de vid sin Oidium $\mathrm{Ni}$ Trichoderma

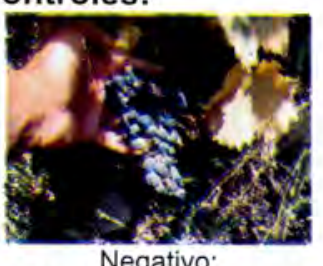

Negativo: Racimo de vid con Oidium sin Trichoderma

\section{Tratamientos Experimentales}

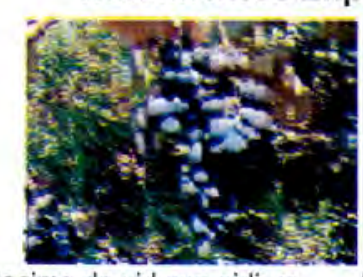

Racimo de vid con oidium

+ Trichoderma sp. TL

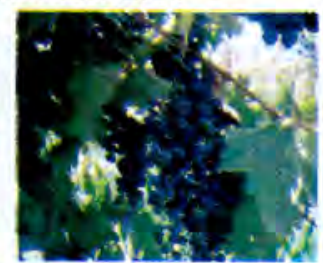

Racimo de vid con Oidium + Trichoderma harzianum 